\title{
Switching and instabilities of optical vortices in nonlinear dual-core photonic crystal fibre couplers
}

José R. Salgueiro

Humberto Michinel

\section{Albert Ferrando}

Yuri S. Kivshar
Departamento de Física Aplicada, Universidade de Vigo, Facultade de Ciencias de Ourense, E-32004 Ourense, Spain

Departamento de Física Aplicada, Universidade de Vigo, Facultade de Ciencias de Ourense, E-32004 Ourense, Spain

Interdisciplinary Modeling Group, Intertech. Departament d'Òptica, Universitat de València. Dr Moliner, 50E-46100 Burjassot (València), Spain

Nonlinear Physics Centre and Centre for Ultrahigh-bandwidth Devices for Optical Systems (CUDOS), Research School of Physical Sciences and Engineering, Australian National University, Canberra ACT 0200, Australia

We study switching of an optical vortex launched into one core of a dual-core waveguide coupler in a photonic crystal fibre with selffocusing nonlinearity. We analyse how the beam power and the angular momentum associated with the vortex mode transfer to the second core of the coupler in both linear and nonlinear regimes. We describe three major scenarios of the vortex dynamics and reveal novel symmetry-breaking instabilities associated with the vortex nonzero angular momentum. [D0I: 10.2971/je0s.2006.06014]

Keywords: Photonic crystal fibres, nonlinear couplers, vortices, switching, angular momentum

\section{Introd uction}

Optical vortices are fundamental localised structures associated with the points of vanishing intensity and phase singularities of optical beams [1]. Optical vortices are generated experimentally in different types of linear and nonlinear optical media $[1,2]$. However, when a vortex beam propagates in a self-focusing nonlinear medium, it becomes unstable due to the symmetry-breaking azimuthal instability [2], and it decays into several fundamental solitons [3]. This vortex azimuthal instability can be suppressed when the vortex beam is placed in a waveguide created in a photonic crystal fibre (PCF) known to support stable fundamental and vortex nonlinear modes $[4,5]$. In strongly nonlinear media, PCF vortex solitons still remain stable when they are perturbed only by symmetric (diagonal) perturbations, but they decay into two fundamental solitons when affected by asymmetric (non-diagonal) perturbations. These fundamental solitons, however, remain confined inside the PCF waveguide instead of flying off, and they do not undergo collapse even in the focusing medium $[4,5]$. This is in a sharp contrast with homogeneous and unbounded nonlinear Kerr media where two-dimensional self-trapped beams -spatial solitons- become unstable and undergo the collapse instability [3].

Photonic crystal fibres, cylindrical structures presenting a lattice of holes running parallel to its optical axis, have turned into an active field of research due to its remarkable properties compared with the conventional fibres [6]. Recent theoretical and experimental papers reported the studies and fabrication of dual-core PCF structures for broadband directional coupling or polarisation splitting [7]-[11]. In addition, the sta- bility of nonlinear modes of a dual-core PCF coupler recently analysed in Ref. [12] allows to describe the basic features of nonlinear switching in the PCF couplers, and the first experimental results for nonlinear switching realised in a dual-core PCF have been reported recently [13].

The purpose of this paper is twofold. First, we study the basic operation of the nonlinear dual-core PCF coupler when, instead of the fundamental mode usually employed for switching of linear and nonlinear couplers, we launch a vortex beam into one core of the coupler. We are interested how a nonzero angular momentum associated with the vortex beam can switch between the cores of the coupler, in both linear and nonlinear regimes, and how the vortex behaves when the rotational symmetry of the structure is destroyed. Generally speaking, we wonder how the "angular momentum switching" of the vortex correlates with the familiar switching observed for the beam power. To the best of our knowledge, this question has never been addressed before, but it seems very important for suggesting novel ways to manipulate, transform and control the angular momentum of light. Second, we study novel types of instabilities of the vortex beam observed during its tunnelling between the cores of the PCF coupler.

The paper is organised as follows. In section 2 we formulate our problem and introduce the basic model and equations which we then solve numerically. Then, in section 3, we summarise our numerical results for the vortex dynamics and describe three different scenarios of the vortex switching, for both linear and nonlinear regimes. 


\section{MODELAND THE BASIC EQUA- TIO N S}

We consider a nonlinear PCF constituted by a triangular lattice of circular holes filled with the dielectric material with the linear refractive index $n_{a}$ in a substrate with the refractive index $n_{s}$ made of a material possessing a nonlinear response. We assume that a dual-core coupler in this PCF is created by two missing holes which are separated at least by another hole or by an interspace between two holes. This restriction seems important because two consecutive PCF defects do not lead to an optical coupler device but instead creates an asymmetric waveguide that supports elliptical modes, as discussed in detail in Ref. [12].

We describe the field propagation in this coupler device using the standard model $[4,5,12]$,

$$
i \frac{\partial E}{\partial z}+\left(\frac{\partial^{2} E}{\partial x^{2}}+\frac{\partial^{2} E}{\partial y^{2}}\right)+\left(W(x, y)+V(x, y)|E|^{2}\right) E=0,
$$

where $E$ is the electric field, $W(x, y)=n_{a}+\delta V(x, y)$, being $\delta=n_{s}-n_{a}$, and the function $V(x, y)$ describes an effective potential created by two missing holes in the perfect PCF lattice (i.e., two solid cores of the coupler). We assume that $V$ takes the value $V=1$ in the substrate, and it vanishes inside the holes, $V=0 . \quad$ In Eq. (1), we have used the nondimensional normalised units, $x, y$ and $z$, related with the dimensional ones, $X, Y$ and $Z$, through a scale factor $\eta$,

$$
\begin{array}{r}
x=X / \eta, \\
y=Y / \eta, \\
z=Z /\left(2 k_{0} n_{0} \eta^{2}\right),
\end{array}
$$

where $k_{0}=2 \pi / \lambda$, being $\lambda$ the vacuum wavelength, and $n_{0}$ is a base refractive index that we choose as the one of the substrate. Additionally, we also considered the index difference $\delta$ a normalised quantity defined by,

$$
\delta=2 k_{0} n_{0} \eta^{2} \Delta
$$

where $\Delta=\tilde{n}_{s}-\tilde{n}_{a}$ is the actual index difference between the substrate $\left(\tilde{n}_{s}\right)$ and the holes $\left(\tilde{n}_{a}\right)$. This normalisation allows us to describe the system independently of the characteristics of the materials -substrate and holes- used for the PCFstructure. In fact, a change in the index difference can always be compensated, accordingly to Eq. (3), by a proper spatial rescaling of the system, i.e. by a proper definition of the scaling factor $\eta$, and the actual dimensions and spatial variables can be easily calculated by means of Eqs. (2). On the other hand, the description can also be made independent of the nonlinear coefficient of the substrate, defining the normalised field as,

$$
E=\left(2 k_{0}^{2} n_{0} n_{2} \eta^{2}\right)^{1 / 2} \mathcal{E},
$$

where $\mathcal{E}$ is the actual (dimensional) electric field and $n_{2}$ the nonlinear Kerr coefficient. This will suppose working with normalised power units which can be easily converted to the actual ones using Eq. (4) as will be done below.

We chose, in our normalised system, the values $n_{s}=3$ and $n_{a}=1$ for the refractive indices (so that $\delta=2$ ), $\Lambda=5$ for the PCF pitch and $r=2$ for the hole radius. This assures that the single-core waveguide supports the first order mode, what implies a specific relation between the hole radius and pitch. As is well-known, unlike conventional fibres, PCFs present for usual values of the parameters a strongly limited number of modes $[6,14]$, being single-mode for a ratio $r / \Lambda \lesssim 0.4$. We would like to stress that choosing particular materials for the PCF-structure is only a matter of calculating the corresponding scaling parameter $\eta$ for the spatial coordinates. So, as an example, if we consider pure silica for the substrate and air for the holes $\left(\tilde{n}_{s}=n_{0}=1.5, \Delta=0.5\right)$, and $\lambda=1.5 \mu \mathrm{m}$ for the wavelength, from Eq. (3) we obtain the scaling factor $\eta=0.2849 \mu \mathrm{m}$ for the actual system fulfilling the requested characteristics.

We aim to study the switching characteristics of a nonlinear PCF coupler with the input vortex mode carrying a nonvanishing angular momentum. The vortex mode has the wellknown structure, $E(r, \phi)=u(r) \exp (i \phi)$, and we generate it by choosing the input beam with the radial amplitude corresponding to the $\mathrm{LP}_{11}$ mode of a step-index optical fibre-linear combination of the almost degenerated modes $\mathrm{TE}_{01}, \mathrm{TM}_{01}$ and $\mathrm{HE}_{21}$ - and launch it into one of the cores of the PCF coupler. The $\mathrm{LP}_{11}$ mode is chosen for a fibre core with the diameter equal to the PCF pitch in order to assure a reasonable good coupling of the initial field to the PCF core waveguide.

We solve Eq. (1) for the field propagation numerically, employing the standard beam propagation algorithm, and calculate the $z$-component of the angular momentum and the beam power, defined as

$$
\begin{array}{r}
L_{z}=-i \int E^{*}(x, y)\left(x \frac{\partial E}{\partial y}-y \frac{\partial E}{\partial x}\right) d x d y \\
P=\int|E|^{2} d x d y
\end{array}
$$

as functions of the propagation distance $z$, for different values of the input field power.

\section{RESULTS AND DISCUSSIONS}

We observe different scenarios of the vortex switching, and three major types of the vortex dynamics are illustrated in Figure 1, where three simulations for three different input powers are presented. The evolution of the beam power and vortex angular momentum are plotted in Figure 2 for the same three cases, along with the example of the pure linear case. Due to the symmetry-breaking instability induced by the presence of the second core, the vortex breaks up into a dipole structure due to the growing azimuthal perturbations. The two dipole modes present a different coupling rate to the second core, and the light distribution inside the core transforms itself when the beam propagates, adopting the shape of a dipole that flips between the two states and partially recovers the vortex shape at intermediate positions between both dipole states. 

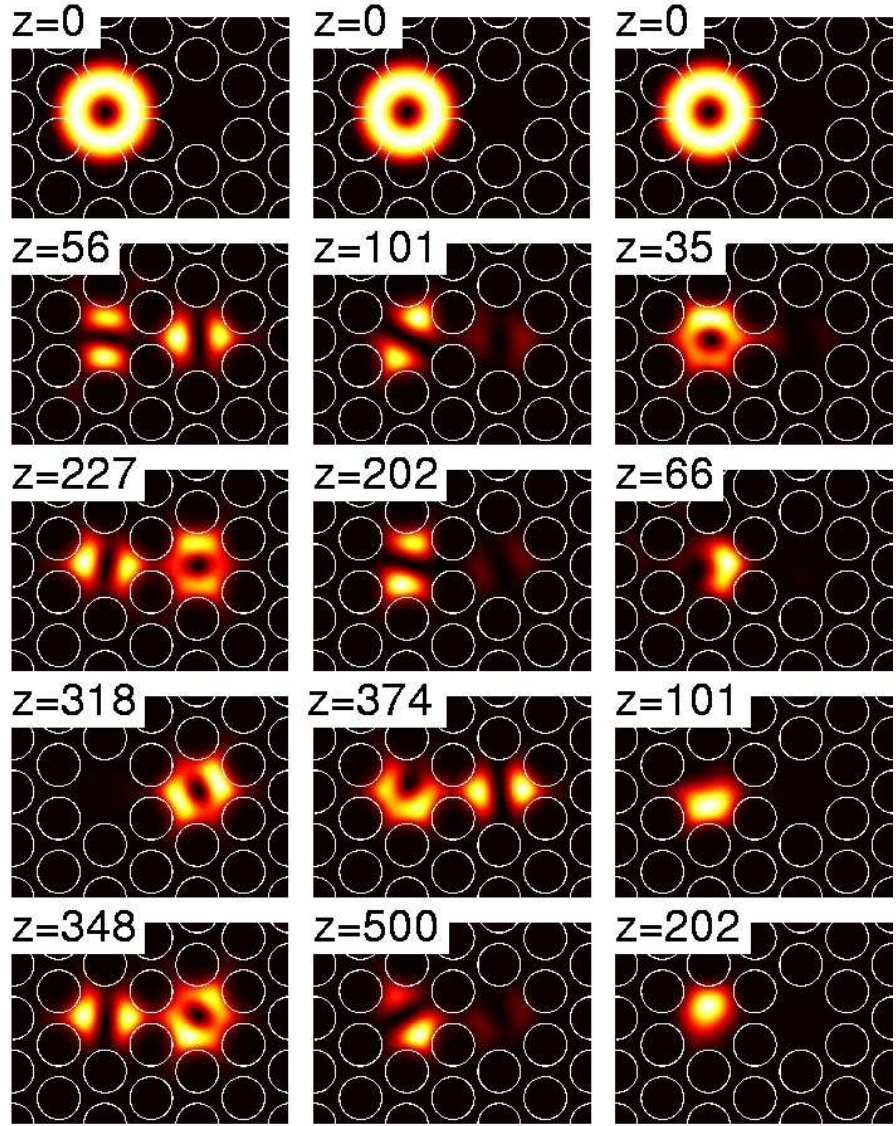

(a)

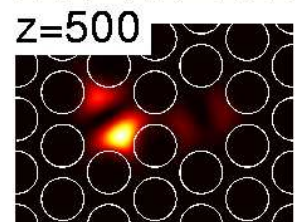

(b)
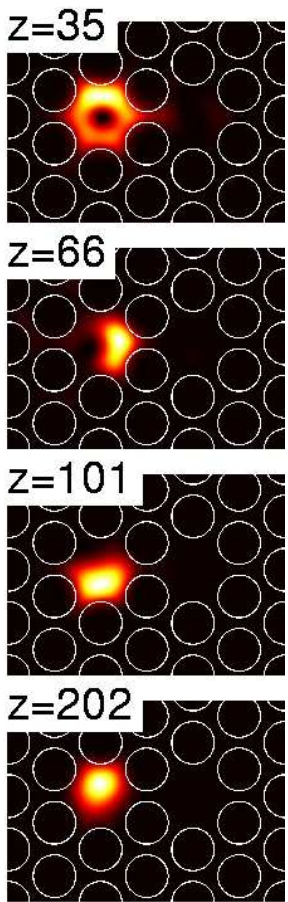

(c)

FIG. 1 Evolution of a vortex beam launched into one core of the nonlinear PCF coupler for different input powers. (a): case I $(P=0.5)$, (b) case II $(P=3)$, and (c) case III $(P=6)$. Click to watch the movies for case I (1.6 MB), case II (1.6 MB) and case III (1.3 MB).

In the linear regime (i.e. for low input powers), we observe a characteristic periodic power coupling between both the PCF cores as illustrated in Figure 2(a). The difference with the switching of a fundamental mode is that in the vortex case we observe relative minima between the absolute ones. Additionally, the absolute ones do not reach a full zero value. This is due to the existence of the two superposed dipole modes which present a different coupling length as discussed above. On the other hand, some amount of the angular momentum is also transferred to the second core and the field emerging at that core also rotates in a similar way as the field in the first core. The angular momentum $L_{z}$ goes through zero at the points where the power reaches a minimum and reaches maxima (in absolute value) at those where power is maximum.

In the nonlinear regime, we observe several different scenarios of the nonlinear switching and instabilities. When the input power is low [case I in Figure 1(a) and Figure 2(b)], the beam dynamics is similar to the linear case, except but minor differences [compare Figure 2(a) and Figure 2(b)] which can be explained by the fact that nonlinearity changes the coupling length of the dipole modes.

When the input beam power raises [see Figure 1(a-b) and Figures 2(c-d), the cases II and III], two different effects are observed. First, the coupling between the PCF cores is progres-

sively suppressed in a similar way as in the familiar case of the fundamental mode switching. Second, there is an increase of the power converted into the fundamental mode. For moderate input powers, the coupling is only partially suppressed [see Figure 2(c)] whereas for high enough input powers all the energy remains in the first core [see Figure 2(d)]. On the other hand, in our simulations we observe that when the input power raises there is an increase of the energy initially radiated that lowers the power in the first core. The remaining power progressively couples to the fundamental mode inside the same core and the vortex structure decays. The angular momentum transfer also decreases for larger powers, and it undergoes a change of the regime when the energy conversion to the fundamental mode starts. In this way, as the beam propagates a superposed higher-frequency component appears, and this corresponds to an orbital angular momentum transferred to the fundamental mode [see Figures 2(cd)]. The original angular momentum component of the vortex progressively disappears, only remaining, after a certain propagation distance, the fraction transferred to the fundamental mode. The amplitude of the oscillations slowly damps, and it eventually vanishes for long propagation distances, resulting in the generation of a fundamental soliton at the centre of the core. The higher oscillation frequency of the orbital angular momentum component could be explained by the fact that the fundamental mode has the power more concentrated at the centre, and this implies a lower momentum of inertia of the beam.
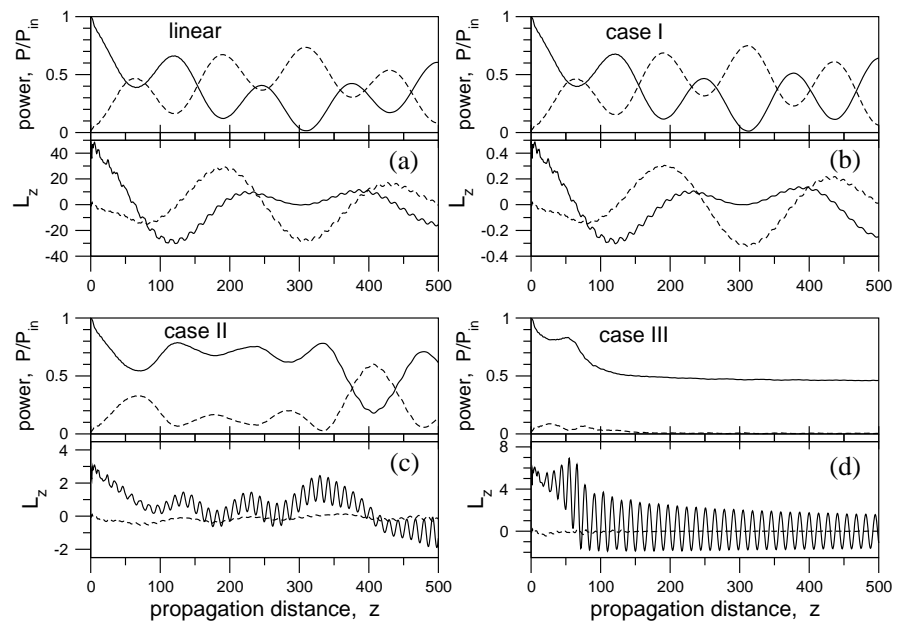

FIG. 2 Power (normalised) and angular momentum $L_{z}$ versus propagation distance for each of the cores of the PCF coupler, when a vortex is launched into one of the cores. The continuous line corresponds to the initially excited core (first core) and the dashed line to the second core. (a) Linear propagation regime. (b-d) Nonlinear regime for different input powers corresponding to the simulations showed in Figure 1 (labels I, II and III are correspondent in both figures). Input powers: $P_{I}=0.5, P_{I I}=3$ and $P_{\text {III }}=6$.

The switching curve for the beam power is presented in Figure 3(a) where it is shown, for the exited core, the ratio between the output and input powers as a function of the input power. We present the results for the vortex input beam as well as for a fundamental beam for the shake of comparison. To build this switching curves, the propagation distance is set at the value corresponding to the first absolute power minimum for the linear propagation regime. For the parameters considered it is 
$z=310.50$, for the vortex, and $z=358.65$, for the fundamental beam. The curve corresponding to the fundamental mode illustrates the well-known nonlinear switching [12], fast raising from zero to almost the unity and then stabilising after some transitory small oscillations. The high-slope part corresponds to the nonlinearity-induced coupling suppression, and it constitutes the key of the nonlinear switching operation. For the vortex input beam, the switching curve is qualitative similar, but two main differences are observed. First, the switching is produced for higher input powers and the transitory regime is more steady. Second, the final power is lower, reaching about half the power as for the fundamental state.
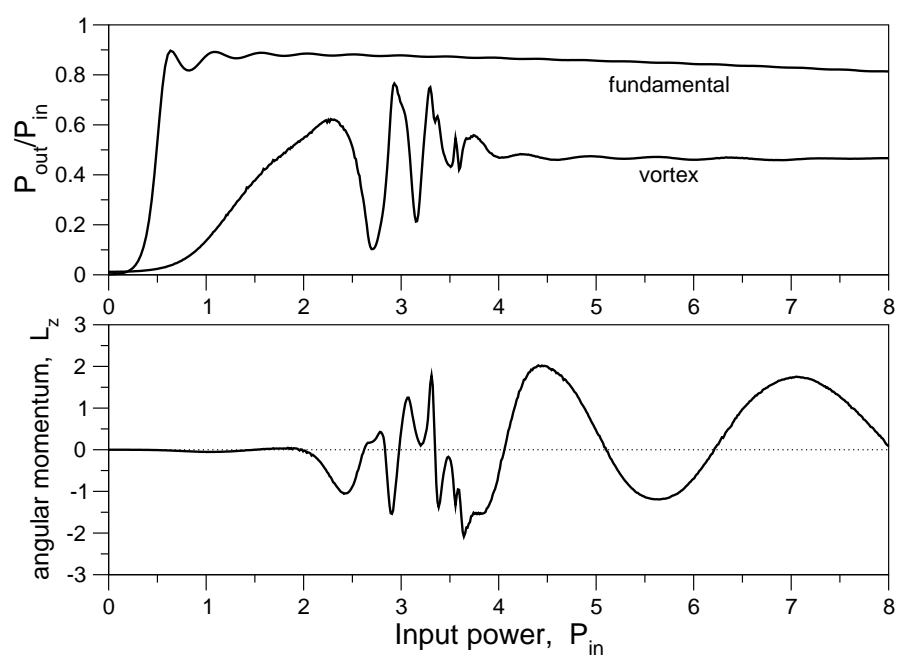

FIG. 3 Switching curves for the dual-core PCF coupler. (a) Power switching when one of the cores is excited by a fundamental $L_{01}$ mode and a vortex made from the two degenerated $\mathrm{LP}_{11}$ dipoles. (b) Angular momentum switching curve for the vortex.

A simple explanation for the higher switching power required for the vortex beam switching is based on the fact that in the case of the vortex the power is distributed all over the ring or it is concentrated in two lobes of the dipoles. Besides, when the input power increases an initial radiation of energy is observed due to the excitation of the fundamental mode as discussed above, lowering the power that remains in the core. This initial energy radiation is also the reason that the final relative power is much lower in the vortex case. On the other hand, the existence of the two dipole modes with different coupling ratios, due to the asymmetry induced by the presence of the second core and due to the nonlinear effect, produces a more complicated and longer transitory state. This switching at different powers for the fundamental and vortex states, together with the fact that the beating length is similar in both cases suggests the possible use of such a device for double switching operations.

We also analyse how the light angular momentum couples between the two cores, and the corresponding switching curve for the angular momentum is shown in Figure 3(b). As follows from those results, the angular momentum remains zero in the second core for low input powers since $L_{z}$ vanishes at the minima of the coupling [see Figure 2(a-b)]. When the input power grows, the momentum undergoes through a transitory state, in a similar fashion to the power curve [see Figure 3(a)], and finally oscillate at high values of the input power as shown in Figure 3(b).

Finally, in order to estimate the actual power $\tilde{P}$ necessary to achieve those effects, in terms of the normalised power $P$, we only have to take into account the normalisation given by Eqs. (2) and (4), so that,

$$
\tilde{P}=\frac{\epsilon_{0} c}{2} \int|\mathcal{E}|^{2} d X d Y=\frac{1}{2 k_{0}^{2} n_{0} n_{2} \eta^{2}} \cdot \int|E|^{2} \eta^{2} d x d y,
$$

being $\epsilon_{0}$ the vacuum dielectric constant and $c$ the speed of light. Taking into account the nonlinear coefficient, $n_{2}^{\prime}$, expressed in $\mathrm{cm}^{2} / \mathrm{W}$, so that $n_{2}=\left(\epsilon_{0} c / 2\right) n_{2}^{\prime}$, we finally obtain,

$$
\tilde{P}=\frac{P}{2 k_{0}^{2} n_{0} n_{2}^{\prime}} .
$$

As an example, for pure silica, taking $\lambda=1.55 \mu \mathrm{m}, n_{2}^{\prime}=$ $2.5 \times 10^{-16} \mathrm{~cm}^{2} / \mathrm{W}$ and assuming $n_{0}=1.5$, we obtain for the normalised power unit $(\mathrm{P}=1)$ in Figure $3, \tilde{P}=8.11 \times 10^{5}$ $\mathrm{W} \approx 800 \mathrm{~kW}$. For a higher nonlinear material, such as $\mathrm{As}_{2} \mathrm{Se}_{3}-$ chalcogenide glass, we take $\lambda=1.55 \mu \mathrm{m}, n_{2}^{\prime}=1.1 \times$ $10^{-13} \mathrm{~cm}^{2} / \mathrm{W}, n_{0}=2.76$ (see Ref. [15]), to obtain $\tilde{P}=10^{3}$ $\mathrm{W}=1 \mathrm{~kW}$. This value is between two and three magnitude orders below the one obtained for pure silica and though it is too high for CW operation, it would be suitable for using a nanosecond-pulse light source.

\section{C O N C L USIONS}

We have analysed linear and nonlinear switching of an optical vortex in a dual-core nonlinear directional coupler created by two neighbouring missing holes in a photonic crystal fibre. We have considered the case when the optical vortex is launched into one core of the coupler and analysed different regimes of the vortex evolution, including vortex switching and nonlinearity-induced instability. We have described three major scenarios of the vortex dynamics and studied in detail the symmetry-breaking instabilities associated with the nonzero angular momentum and the vortex tunnelling to the second core of the coupler, in both linear and nonlinear regimes.

\section{A C K N O W LED GEMEN TS}

JRS acknowledges a Ramón y Cajal contract granted by the Secretaría de Estado de Universidades e Investigación of Spain. Also JRS and HM acknowledge support from MEC (Spain), through the projects FIS200402466 and FIS2004-20188-E, and Xunta de Galicia (project PGIDIT04TIC383001PR). AF acknowledges support from MEC (Spain), project FIS2005-01189. YSK acknowledges a support of the Australian Research Council and thanks Anton Desyatnikov for useful discussions and suggestions.

\section{References}

[1] See for example M. S. Soskin and M. V. Vasnetsov, "Optical vortices," in Progress in Optics, E. Wolf, ed., Vol. 42, p. 219 (NorthHolland, Amsterdam, 2001) and references therein. 
[2] A. S. Desyatnikov, Yu. S. Kivshar, and L. Torner, "Optical vortices and vortex solitons" in Progress in Optics, E. Wolf, ed., Vol. 47, pp. 291-391 (North-Holland, Amsterdam, 2005).

[3] See, e.g., Yu.S. Kivshar and G. Agrawal, Optical Solitons: From Fibers to Photonic Crystals (Academic Press, San Diego, 2003), Sec. 6.5 and references therein.

[4] A. Ferrando, M. Zacarés, P. Fernandez de Córdoba, D. Binosi, and J.A. Monsoriu, "Spatial soliton formation in photonic crystal fibers" Opt. Express 11, 452-459 (2003).

[5] A. Ferrando, M. Zacarés, P. Fernandez de Córdoba, D. Binosi, and J.A. Monsoriu, "Vortex solitons in photonic crystal fibers", opt. Express 12 817-822 (2004).

[6] P. St. J. Russell, "Photonic crystal fibers" Science 299, 358-362 (2003).

[7] See, for example, F. Fogli, L. Saccomandi, P. Bassi, G. Bellanca, and S. Trillo, "Full vectorial BPM modeling of index-guiding photonic crystal fibers and couplers" Opt. Express 10, 54-59 (2002).

[8] L. Zhang and Ch. Yang, "Polarization splitter based on photonic crystal fibers" Opt. Express 11, 1015-1020 (2003).

[9] K. Saitoh, Y. Sato, and M. Koshiba, "Coupling characteristics of dual-core photonic crystal fiber couplers" Opt. Express 11, 31883195 (2003).

[10] H. Kim, J. Kim, U.-C. Paek, B.H. Lee, and K.T. Kim, “Tunable photonic crystal fiber coupler based on a side-polishing technique" Opt. Lett. 29, 1194-1196 (2004).

[11] J. Lagsgaard, 0. Bang, and A. Bjarklev, "Photonic crystal fiber design for broadband directional coupling" Opt. Lett. 29, 2473-2475 (2004).

[12] J.R. Salgueiro and Yu. S. Kivshar, "Nonlinear dual-core photonic crystal fiber couplers" Opt. Lett. 30, 1858-1860 (2005).

[13] A. Betlej, S. Suntsov, K.G. Makris, L. Jankovic, D. N. Christodoulides, G. I. Stegeman, J. Fini, R. T. Bise, and D. J. DiGiovanni, "All-optical switching and multifrequency generation in a dual-core photonic crystal fiber" Opt. Lett. 31, 1480-1482 (2006).

[14] A. Ferrando, E. Silvestre, J.J. Miret, P. Andrés, "Vector description of higher-order modes in photonic crystal fibers" J. Opt. Soc. Am. A 17, 1333-1340 (2000).

[15] I.D. Chremmos, G. Kakarantzas, N.K. Unzunoglu, "Modeling of a highly nonlinear chalcogenide dual-core photonic crystal fiber coupler" Opt. Comm. 251, 390-393 (2004). 